\title{
Comparison of diagnosis of temporomandibular joint disorders based on RDC/TMD Axis I and DC/TMD Axis I
}

\author{
${ }^{1}$ Brigitta Swasti Lestari, ${ }^{2}$ Rasmi Rikmasari, ${ }^{2}$ Setyawan Bonifacius \\ ${ }^{1}$ Profession Program Student \\ ${ }^{2}$ Department of Prosthodontics \\ Faculty of Dentistry, Padjadjaran University \\ Bandung, Indonesia \\ Corresponding author: Rasmi Rikmasari, email: rasmi.rikmasari@fkg.unpad.ac.id
}

\begin{abstract}
Introduction: The Research Diagnostic Criteria for Temporomandibular Disorders (RDC/TMD) has become the most widely used diagnostic protocol for research in temporomandibular disorders (TMD). The invalidity of RDC/ TMD in clinical application causes the revision of RDC/TMD to be the Diagnostic Criteria for Temporomandibular Disorder(DC/TMD). The purpose of this study was to determine the differences in diagnosis of TMD between RDC/ TMD examination and DC/TMD Axis I on students of Faculty of Dentistry, Padjadjaran University. Method: The type of this research was comparative survey approach using clinical examinations and questionnaires. The sample was collected from 48 people using random sampling techniques. The diagnosis of TMD was obtained by filling in the symptom questionnaire and clinical examination based on RDC/TMD Axis I and DC/TMD Axis I, which is then entered into the RDC/TMD diagnosis algorithm and DC/TMD decision tree. Results: The results showed that from 48 samples there were $36(75 \%)$ people with the same diagnosis of RDC/TMD and DC/TMD, and 12 (25\%) people with different diagnoses between RDC/TMD and DC/TMD. Conclusions: Based on the results of the study, the diagnosis of TMD based on RDC/TMD were still categorized the same as the diagnosis based on DC/TMD.
\end{abstract}

Keywords: temporomandibular disorders, Research Diagnostic Criteria Axis I

\section{INTRODUCTION}

The temporomandibular joint (TMJ) is a joint thatconnects the mandible or lower jaw tothe skull and regulates jawmovement; ${ }^{1}$ one of the mostcomplex, vulnerable, and highly usedjoints in themovement of the humanbody. ${ }^{2}$ Temporomandibularjoint disorders (TMDs) are a term commonly used for problems related to the jaw joint, involving the muscles of mastication, the TMJ and related structures or both. ${ }^{3}$ The prevalence of TMD is $40-60 \%$ of the world's population. ${ }^{4}$ The TMDs are most common in people ages 20-40years, and is more common in women than in men. ${ }^{5}$

The Research Diagnostic Criteria for Temporomandibular Disorders (RDC/TMD)has beenthe most widely used diagnostic protocol for the research of TMDs since its publication in $1992 .{ }^{6}$ Then in 2010, a revised RDC/TMDwas developed. ${ }^{7}$ Diagnosis of RDC/TMD Axis I did not reach the target set at sensitivity 0.70 and specificity $0.95 .{ }^{\circ}$ The invalidity of RDC/TMD in clinical application led to revision of RDC/TMD to become Diagnostic Criteria for TemporomandibularDisorder (DC/TMD). ${ }^{9}$ The $\mathrm{DC} / \mathrm{TMD}$ provides a comprehensive assessment of the mostcommon TMDs, based on a biopsychosocial model of chronic pain. ${ }^{6}$

The two main goals of $\mathrm{DC} / \mathrm{TMD}$ are to improve and standardize diagnostic groups for further research on TMDs, and to improve clinical care..$^{10}$ The differences in clinical examination procedures for
RDC/TMDandDC/TMDare in the location of pain, static landmarks, mobility, TMJ examination, palpation of muscles and TMJ. ${ }^{11}$

The DC/TMD also consists of two axises, nameIy Axis I which is the procedure for clinical examination, and Axis II which is a questionnaire of disease history and psychological factors. ${ }^{6}$ Axis IDC/ TMD protocol is a very specific and reliable diagnostic criteria that includes valid screening for the detection of pain-associated TMDs as well as valid diagnostic criteriafor distinguishing those most associated with pain from TMDs (sensitivity 0.86 , specificity 0.98 ) and intra-articular disturbances (sensitivity 0.80 and specificity 0.97 ). The Axis II protocol retains the original $R D C / T M D$ screening instrument plus a new instrument for assessing jaw function and additional behaviorand psychosocial factors. ${ }^{6}$ Based on the above background, this study aims to see if there are differences in the diagnosis of TMDs based on RDC/TMD andDC/TMDexaminations.

\section{METHODS}

This type of research is a comparative survey approach using clinical examination and questionnaires. The population of the study was preclinical students of the 2015Faculty of Dentistry, Padjadjaran University as many as 186 people. Sampling employed random sampling technique. ${ }^{12}$ According to Fraenkel and Wallen ${ }^{13}$, the minimum number 
of samples for this type of causal-comparison research was 30 .

The inclusion criteria in this study were preclinical students class of 2015 Faculty of Dentistry, University of Padjadjaran who were willing to participate in this study. Exclusion criteria were 1)moderate or have performed TMJ treatment, 2) has experienced trauma to the TMJ within 2 months, 3) currently taking non-steroidal anti-inflammatory drugs, and 4) currently under dental treatment.

Tools and materials used 1) basicexamination tools; 2 ) informed consent as the respondent's consent form to participate in this study; (3)Axis I clinical examination questionnaire, 2010 Revised RDC/ TMD diagnosis algorithm, Axis I clinical examination questionnaire, and DC/TMD decisiontree diagnosis; and (4) a sheet of recapitulation of examination data on the TMJ.

The procedureswere 1)submitting ethical clearance to the Ethics Committee of Padjadjaran University; 2) calibrating the RDC/TMD and DC/TMD Axis I examination; 3 ) screening students who met predetermined criteria; 4) preparing tools and materials to be used in research;5) explaining to the respondent what would be done and the objectives to be achieved; 6 ) having understood and was willing to follow all research procedures, the respondent expressed his/heragreement by signing an informed consentform;7) recording the identity data of the respondents who have filled out the approval letter; 8) examining TMD with RDC/TMD, followed by DC/TMD examination; 9) recording the data obtained in the examination sheet and then processed them using the 2010 revised RDC/TMD algorithm and DC/TMD decision tree.

\section{RESULTS}

This research was conducted from November to December 2018 at the Faculty ofDentistry, Universitas Padjadjaran Jatinangor after receiving an ethical clearence letter No. 2/7/UN6.KEP/EC/2018 from the Health Research Ethics Commission of Universitas Padjadjaran. The number of samples were 48 students who were selected based on predetermined criteria. Table 1 shows the distribution of the sample by sexandage; the number of sampleswere 39 women $(83.2 \%)$ and 9 men (16.8\%). Majority of the samples in this study were women because the population of preclinical students from the 2015 were mostly women.

Table 1 Distribution of samples by gender and age

\begin{tabular}{|c|c|c|c|c|c|c|}
\hline \multirow{2}{*}{ Variable } & \multicolumn{6}{|c|}{ Category } \\
\hline & \multicolumn{3}{|c|}{ Male } & \multicolumn{2}{|c|}{ Female } & Total \\
\hline \multirow[t]{2}{*}{ Gender } & \multicolumn{3}{|c|}{9} & \multicolumn{2}{|c|}{39} & 48 \\
\hline & \multicolumn{3}{|c|}{$18.8 \%$} & \multicolumn{2}{|c|}{$81.2 \%$} & $100 \%$ \\
\hline \multirow{3}{*}{ Age } & 20 & 21 & 22 & 23 & 34 & \\
\hline & 2 & 37 & 7 & 1 & 1 & 48 \\
\hline & $4.2 \%$ & $77 \%$ & $14.6 \%$ & 2.1 & 2.1 & 100 \\
\hline
\end{tabular}

From the 48 research samples, the results of TMD diagnosis based on the 2010 Revised RDC/ TMD and TMD/DC are seen at Table 2; the most TMD diagnoses based on RDC/TMD identified were disc displacement with reduction (27\%), then myofascial pain (6.3\%), and a combination of myofascial pain and disc displacement with reduction $(6.3 \%)$. In 23 people (47.9\%)none of the diagnoses were found. The most TMD diagnoses based on DC/TMD identified were disc displacement with reduction $(27.1 \%)$, then the combination of myofascial pain and disc displacement with reduction as many $6.3 \%$, in 21 people $(43.75 \%)$ found none of the diagnoses.

From the results of the TMD diagnosis based on the 2010 Revised RDC/TMD and DC/TMD, conclusions were drawnabout the similarities and

Table 2 Results of TMD diagnosis based on 2010 Revised RDC/TMD and DC/TMD Axis I

\begin{tabular}{|c|c|c|c|c|}
\hline \multirow{2}{*}{ Diagnosis Results } & \multicolumn{2}{|c|}{ RDC-TMD } & \multicolumn{2}{|c|}{ DC-TMD } \\
\hline & $\mathbf{f}$ & $\%$ & $\mathbf{f}$ & $\%$ \\
\hline la Myofascial Pain & 3 & 6.3 & 1 & 2.1 \\
\hline Ib Myofascial Pain with Limited Opening & 1 & 2.1 & 1 & 2.1 \\
\hline Ila Disc Displacement with Reduction & 13 & 27.0 & 0 & 0.0 \\
\hline Ilb Disc Displacement without Reduction with Limited Opening & 0 & 0.0 & 1 & 2.1 \\
\hline Ilc Disc Displacement without Reduction without Limited Opening & 0 & 0.0 & 2 & 4.2 \\
\hline Illa Arthralgia & 1 & 2.1 & 13 & 27.1 \\
\hline IIlb Osteoarthritis & 0 & 0.0 & 0 & 0.0 \\
\hline Illc Osteoarthrosis & 2 & 4.2 & 0 & 0.0 \\
\hline la, Ila, IIla & 1 & 2.1 & - & - \\
\hline la, Ila & 3 & 6.3 & 2 & 4.2 \\
\hline la, Ila, Ilb & 1 & 2.1 & - & - \\
\hline Id, Ila & - & - & 2 & 4.2 \\
\hline le, llc & - & - & 1 & 2.1 \\
\hline None & 23 & 47.9 & 3 & 6.3 \\
\hline Total & 48 & 100.0 & 48 & 100.0 \\
\hline
\end{tabular}


Table 3 Comparison of TMD diagnosis results based on RDC/TMD and DC/TMD Axis

\begin{tabular}{lccc}
\hline Comparison of TMD Diagnosis & $\mathbf{f}$ & $\%$ & p-value \\
\hline The same results of the diagnosis & 36 & 75 & \\
Disc displacement with reduction & 11 & 22.9 & \\
Myofascial pain & 1 & 2.1 & \\
Arthralgia & 1 & 2.1 & 0.0966 \\
Degenerative joint diseases & 2 & 4.2 & \\
No diagnosis found & 21 & 43.7 & \\
Different diagnostic results & 12 & $25 \%$ & \\
\hline
\end{tabular}

Note: $p$-value is obtained from the results of the Wilcoxon-Mann/Whitney Test

and differences between the two methods. In Table 3,36 people $(75 \%)$ had the same diagnosis, while 12 people $(25 \%)$ had different diagnosis. The findings of the diagnosis using the two methods were then analyzed using the one sample WilcoxonMann/Whitneytest method. Based on the results of hypothesis testing with the Wilcoxon-Mann/ Whitney test, a p-value of 0.096 was produced. This value was greater than the 0.05 significance level, so the conclusion is that there was no difference in the diagnosis results between RDC/TMD and DC/TMD Axis I.

\section{DISCUSSION}

Several studies have shown that TMDs were more common in women than in men. ${ }^{5,14,15}$ This may be due to hormonal factors which are one of the factors causing TMDs. The TMDs have been linked to the female hormones that disrupt the pain threshold. According to Menezes ${ }^{16}$, women's estrogen levels may lead to higher joint tissue tenderness, resulting in a lower ability to withstand functional stress. However, this contradicts Gray et al. ${ }^{17}$ which claims based on an epidemiological survey, the number of women and men with TMDs showed almost the same results in the population.

The same diagnosis between RDC/TMD and $\mathrm{DC} / \mathrm{TMD}$ was myofascial pain, disc displacement with reduction, disc displacement without reduction with limited opening, disc displacement without reduction without limited opening, arthralgia, and osteoarthritisalong with osteoarthrosis or degenerative joint diseases. The only diagnosis on RDC/ TMD and not on DC/TMD is myofascial pain with limited opening. Meanwhile, the only diagnoses in DC/TMD and no RDC/TMD were local myalgia, myofascial pain with referral, headache attributed to TMD, and disc displacement with reduction with intermittent locking.

The diagnosis of RDC/TMD Group II was disc displacement divided into right and leftjoints. However, for testing the hypothesis, it is not differentiated so that it equates with the diagnosis of DC/ TMD which is not distinguished from right or left.
Thus, if the respondent has disc displacement in one joint, disc displacement is detected. The diagnosis of RDC/TMD of osteoarthritis and osteoarthrosis is also equated with the diagnosis of $\mathrm{DC} /$ TMD of degenerative joint diseases, because osteoarthritis and osteoarthrosis represent a subdiagnosis of degenerative joint diseases hypothesis is not distinguished.

Based on Table2, the diagnosis for RDC/TMD Group I were 9 people and DC/TMD were 10 people. To diagnose myofascial pain in RDC/TMD, it is necessary to have pain in at least 3 of the 20 areas of muscle palpation. ${ }^{18}$ The RDC/TMD diagnosticalgorithm for Group I is simpler than DC/TMD because there are only 2 subdiagnosis, while on DC/ TMD there are 5 subdiagnosis. Five subdiagnoses in DC/TMD Group I made the decision tree diagnosis in Group I more complicated than RDC/ TMD.

From the results of the diagnosis of Group I, there was only 1 person who had the samediagnosis, namely myofascial pain. The discrepancy was attributable to the greater number of subdiagnoses in DC/TMDGroup I.Newdiagnoses in DC/TMD Group I, namely local myalgia, myofascial pain with referral, and headache attributed to TMD led to a significant difference in the diagnostic results in Group I diagnoses. Headache attributed to TMD was added to DC/TMD because there is an increasing in evidence that several forms of headache can occur in association with TMD. ${ }^{19}$ The differential diagnosis in this study was most pronounced in Group I. The difference in detecting local myalgia and myofascial pain was that in local myalgia the absence of pain that extends beyond the palpated area, as opposed to myofascial pain.

The DC/TMDcan be said to be more sensitive in diagnosing Group I disorders because there are more subdiagnosis. This is in line with Steenks ${ }^{18}$ and Schiffman et al ${ }^{6}$ which stated that $D C / T M D$ is very pain oriented. However, this is not in line with Look et $\mathrm{a}^{\mathrm{RO}}$ which stated that the RDC/TMD protocol can diagnose myofascial pain well.

Based on Table 2, the most common diagnosis 
from this study was disc displacement with reduction. The prevalence of disc displacement with reduction increases with age, $6 \%$ in childhood, about $34 \%$ in adolescents, and $31-34 \%$ in adulthood. ${ }^{21}$ According to Farrar and McCarty 22 , nearly $70 \%$ of patients with TMDs experience disc displacement. In this study, 18 samples detected with disc displacement with reduction, 18 people in RDC/TMD and 17 people in DC/TMD. One of the symptoms of disc displacement with reduction is an abnormal mouth opening pattern, namely deviation. ${ }^{23}$

All samples that experienced disc displacement with reduction experienced clicking symptoms. In most cases, $70-80 \%$ TMJ clicking sound is caused by disc displacement in various directions, but mostly in anteromedial direction. ${ }^{24}$ Abnormalities in joint structure and function, such as deformation of joint structures, and changes in synovial fluid quality lead to jointsounds on temporomandibular. This abnormality causes increased friction between the joint elements, resulting in joint sound. ${ }^{25}$

The most samples with the same diagnosis between RDC/TMDand DC/TMD was disc displacement with reduction so that it can be said that in diagnosing this disease, $\mathrm{RDC} / \mathrm{TMD}$ is still reliable. This is in line with Look et $a^{20}$ and Lausten et $a^{26}$, which stated that the reliability of the RDC/TMD protocol can be trusted for the diagnosis of myofascial pain, arthralgia, disc displacement with reduction, and disc displacement without reduction with limited opening. However, what distinguishes between RDC/TMD and DC/TMD is the calculation of the RDC/TMD diagnosis algorithm which is divided into left and right joints.

Samples diagnosed with disc displacement without reduction without limited opening on $\mathrm{DC} /$ TMD were not diagnosed with RDC/TMD. This is in line with Look et $a^{20}$ which stated that the reliability of RDC/TMD for disc displacement withoutreduction without limited opening and osteoarthrosis was unreliable.

The undiagnosed sample with disc displacement without reduction without limited opening was caused by the calculation of the RDC/TMD diagnosis algorithm which included other combinations, namely Maximum Assisted Opening and Passive Stretch, namely Max $35 \mathrm{~mm}$ and Stretch $4 \mathrm{~mm}$. This does not result in any diagnosis in the $\mathrm{RDC} / \mathrm{TMD}$ diagnosis algorithm. The DC/TMD decision tree diagnosis is simpler in diagnosing disc displacement without reduction without limited opening because there is only a MaximumAssisted Opening requirement of $40 \mathrm{~mm}$. This diagnosis in
DC/TMDalso does not take into account whether the sample has clicked during opening, closing the mouth, lateral movement, and protrusion movement as in RDC/TMD.

Degenerative joint disorders are confirmed by the presence of joint crepitus sounds. ${ }^{27}$ Osteoarthrosis is a subdiagnosis of degenerative joint diseases in DC/TMD Group III. From this study, there were 2 samples with osteoarthritis in RDC/TMD Group III, and degenerative joint diseases. This is not in line with Look et $a^{20}$ saying that RDC/TMD cannot be relied upon in diagnosing osteoarthritis, because samples can still be diagnosed using RDC/TMD examination.

Bernhardtet $a^{R 8}$ found the prevalence of osteoarthritis of the TMJjoint on clinical examination and MRI was $25 \%$ in the $20-49$ years age group. The sample of this study was 20-24 years old, so it was included in the prevalence. Schmitter et $a^{29}$ found that the prevalence of osteoarthritis was $70 \%$ in the 73-75 years age group.

Steenks ${ }^{18}$ said that RDC/TMD tends to result in too many diagnoses leading to overtreatment. This may be due to the RDC/TMD diagnostic algorithm that distinguishes the right and left joints in Groups II and III thereby increasing the possibility of differential diagnosis between the right and left joints. In this study, there was 1 person who had a different diagnosis between the right and leftjoints. Overdiagnosis also occurred in 1 person who was diagnosed with myofascial pain and arthralgia. The occurrence of overdiagnosis due to RDC/TMD arthralgiawas assigned to Group III (other joint diseases), while DC/TMD arthralgia was assigned to Groupl. Thus, it was impossible for a sample to experience more than 1 diagnosis in one group.

Based on Table 2, there were 23 people and 21 samples, respectively, whose diagnosis of TMD was not found. In Table 1, it can be seen that the age range of the sample in this study was 20-24 years. Factors that cause TMDs are psychological factors, such as anxiety and stress, structural factors (occlusion), functional (bruxism), genetic factors, orthodontic treatment and external trauma. ${ }^{30}$ According to Kindler et $a^{\beta 1}$, psychological factors can trigger muscle hyperactivity, followed by biomechanical changes and pain. Psychological factors can also lead to increased production of neurotransmitters and serotonin, catecholamine imbalance, which causes pain, especially pain in the temporomandibular region. This thing shows that the possibility of stress levels in preclinical students from the 2015 Faculty of Dentistry, Universitas Padjadjaran, is not too high. 
In this study, there were 21 people whowere not detect TMDs. This suggests that TMD is less commonamong universitystudents, as in the study of Minghelli, ${ }^{32}$, who evaluated university students in the health sector and found the prevalence of TMD to be only $37.3 \%$. However, this contradidicts the study of Oliveira et a/ ${ }^{33}$ on college students in Brazil, where the prevalence was $68.6 \%$.

Based on Table 2 thereare 48 samples in this study. The samples who had the same diagnosis were 36 people $(75 \%)$ and no diagnosis was found in 21 people. Meanwhile, the other 12 people or $25 \%$ found different diagnosis results. This shows that RDC/TMD and DC/TMD show the samediagnostic results are still more dominant than the re- sults of different diagnoses so that it can be said to be the same. This is in line with Reiter's ${ }^{34}$ statement, that there was no significant difference between RDC/TMD and DC/TMD for Axis I diagnoses, including Group I (muscledisorders), Group II (disc disorders), and Group III (arthralgia, degenerative joint disease). However, this is not in line with the statements which state that $\mathrm{DC} / \mathrm{TMD}$ is more valid than RDC/TMD. $6,35,36$

The conclusion of this study is the diagnosis of TMD based on RDC/TMD is still in the same category as the results of the diagnosis of DC/TMD with a similarity level of $75 \%$ which indicates that the same diagnosis is still more dominant than the results of different diagnoses.

\section{REFERENCES}

1. Tanaka E, Koolstra JH. Biomechanics of the temporomandibular joint. J Dent Res. 2008;87(11):989-91.

2. Alomar X, Medrano J, Cabratosa J, Clavero JA, Lorente M, Serra I, et al. Anatomy of the temporomandibular joint. Semin Ultrasound, CT MRI 2007; 28(3):170-83.

3. Herdiyani I, Kurnikasari E, Damayanti L. The severity of temporomandibular joint disorder by teeth loss in the elderly. Padjadjaran J Dent 2011;23(2):85-8.

4. Ryalat S. Prevalence of temporomandibular joint disorders among students of the University of Jordan. J Clin Med Res 2009;1(3):158-64.

5. Ingawalé S, Goswami T. Temporomandibular joint: disorders, treatments, and biomechanics. Ann Biomed Eng 2009;37(5):976-96.

6. Schiffman E, Ohrbach R, Truelove E, Look J, Anderson G, Goulet JP, et al. Diagnostic criteria for temporomandibular disorders (DC/TMD) for clinical and research applications: recommendations of the international RDC/ TMD consortium network and orofacial pain special interest group. J Oral Fac Pain Headache 2014;28(1):6-27

7. List T. Introduction of research diagnostic criteria for temporomandibular disorders (RDC/TMD). 2010. [cited 26 Sep 2018]. Available from: http://www.rdctmdinternational.org/

8. Kaphingst KA, Persky S, Lachance $\mathrm{C}$. Research diagnostic criteria for temporomandibular disorders: validity of axis I diagnoses. J Orofac Pain. 2010;14(4):384-99.

9. Vilanova LSR, Garcia RCMR, List T, Alstergren P. Diagnostic criteria for temporomandibular disorders: selfinstruction or formal training and calibration? J Headache Pain 2015;16(1):1-9.

10.Sessle BJ. Diagnostic criteria for temporomandibular disorders: evolution and debate. J Oral Facial Pain Headache 2018;32(1):5.

11. Ohrbach R. Diagnostic criteria for temporomandibular disorders : assessment instruments. 2016. [cited 9 Mei 2018]. Available from: www.rdc-tmdinternational.org

12. Sugiyono. Metode penelitian. Bandung: Alfabeta; 2003. p.74.

13. Siegel S. Statistik non parametrik untuk ilmu-ilmu sosial. Jakarta: PT Gramedia; 1986. p.100.

14. Detamore MS. Structure and function of the temporomandibular joint disc: implications for tissue engineering. J Oral Maxilofac Surg 2003;61(4):494-506.

15.van Loon J, de Bont LGM, Stegenga B, Spijkervet FKL, Verkerke GJ. Groningen temporomandibular joint prosthesis. Development and first clinical application. J Oral Maxilofac Surg 2002;31(1):44-52.

16. Menezes MS, Bussadori SK, Fernandes KPS, Biasotto-Gonzalez DA. Correlação entre cefaléia e disfunção temporomandibular correlation between headacheand temporomandibularjoint dysfunction. Fisioter e Pesqui 2008;15(2):183-7.

17. Gray R, Davies S, Quayle A. Temporomandibular disorders: a clinical approach. London: British Dental Association; 1995. p.15.

18. Steenks MH, Care SD, Habil D, Wijer A De, Lecturer S, Dentistry P, et al. Reliability and validity of the diagnostic criteria for temporomandibular disorders axis I in clinical and research settings: a critical appraisal. J Oral Facial Pain Headache 2018;32(1):7-18.

19. Leskinen J, Suvinen T, Teerijoki-Oksa T, Kemppainen P, Näpänkangas R, Alstergren $P$, et al. Diagnostic criteria for temporomandibular disorders (DC/TMD): interexaminer reliability of the Finnish version of Axis I clinical diagnoses. J Oral Rehabil 2017;44(7):493-9.

20. LookJ, Wilcox T, HirshkowitzA. Research diagnostic criteria for temporomandibular disorders: reliability of axis I diagnoses and selected clinical measures. J Orofac Pain 2010;24(1):25-34. 
21. Lalue-Sanches M, Gonzaga A, Ribeiro E. Disc displacement with reduction of the temporomandibular joint: the real need for treatment. J Pain Reli 2005;4(5):1-5.

22. Farrar W, McCarty W. The TMJ dilemma. J Ala Dent Assoc 1979;63(1):19-26.

23. Barkin $S$, Weinberg $S$. Internal derangements of the temporomandibular joint: the role of arthroscopic surgery and a rthrocentesis. J Can Dent Assoc 2000;66:199-203.

24. Jerolimov V. Temporomandibular disorders and orofacial pain. Med Sci 2009;33:53-77.

25. Bisi MA, Chaves KD. Relationship between sounds and disc displacement of the temporomandibularjoint using magnetic resonance imaging. 2010;25(1):37-41.

26. Lausten LL, Glaros AG, Williams K. Inter-examiner reliability of physical assessment methods for assessing temporomandibular disorders. Gen Dent 2004;52(6):509-13.

27. Maydana VA, de Souza TR, Denardin VPO, da Silva JUW, Dworkin FS, dos Campos SJP, et al. Possible etiological factors in temporomandibular disorders of articular origin with implications for diagnosis and treatment. Dent Press J Orthod 2010;781515(793):78-8678.

28. Bernhardt O, Biffar R, Kocher T, Meyer G. Prevalence and clinical signs of degenerative temporomandibular joint changes validated by magnetic resonance imaging in a non-patient group. Ann Anat 2007;189(4):342-6

29. Schmitter M, Essig M, Seneadza V, Balke Z, Schröder J, Rammelsberg P. Prevalence of clinical and radiographicsigns of osteoarthrosis of the temporomandibular joint in an older persons community. Dentomaxillofac Radiol 2010;39(4):231-4.

30. Hwang S, Park S. Experience of orthodontic treatment and symptoms of temporomandibular joint in South Korean adults. Iran J Public Health 2018;47(1):13-7.

31. KindlerS, Samietz S, Houshmand M, Grabe HJ, Bernhardt O, Biffar R, et al. Depressive and anxiety symptoms as risk factors for temporomandibular joint pain: A prospective cohort study in the general population. $J$ Pain 2012;13(12):1188-97.

32. Minghelli B, Kiselova L, Pereira C. Associaão entre os sintomas da disfunão temporomandibular com factores psicológicos e alteraões na coluna cervical em alunos da Escola Superior de Saúde Jean Piaget do Algarve. Rev Port Saude Publica [Internet]. 2011;29(2):140-7. Available from: http://dx.doi.org/10.1016/S0870-9025 (11)70018-9

33. de Oliveira AS, Bevilaqua-grossi D, Dias EM. Sinais e sintomas da disfunção temporomandibular nas diferentes regiões brasileiras Signs and symptoms of temporomandibular disorders across Brazilian regions. Rev Bras Fisioter. 2008;15(4):392-6.

34. Reiter S, Eli I, Friedmn-Rubin P. Comparing Axis II scores according to the RDC/TMD and DC/TMD in Israeli patients. J Oral Facial Pain Headache 2017;31(4):323-30.

35. Sessle BJ. Two new entities: newly developed diagnostic criteria for temporomandibular disorders. J Oral Facial Pain Headache. 2013;28(1):5.

36. Truelove EL, WP, Look J. Research diagnostic criteria for temporomandibular disorders: validity of axis I diagnoses. J Orofac Pain 2010;24(1):35-47. 